\title{
母婴分离诱导子代抑有大鼠肠道氮基酸代谢失调
}

张玉荣 ${ }^{1}$, 王瑞忠 ${ }^{2}$, 王 莉 ${ }^{1}$, 陈 莒

1. 西安医学院第一附属医院科研科, 陕西西安 710077

2. 西安大兴医院制剂中心, 陕西西安 710016

[摘要] 目的:探索母婴分离诱导产后子鼠的抑郁样行为及对小肠氨基酸和氨 基酸转运体的影响。方法: 采用母婴分离建立子鼠抑郁模型, 将 SD 鼠随机分为 对照组 $(n=8)$ 和母婴分离组 $(n=8)$ 。对照组母鼠在产后不进行任何千预。母婴分离 组的母鼠在正常分婏后与子鼠连续分离 $14 \mathrm{~d}$, 每天分离 $3 \mathrm{~h}$ 。采用糖水偏好实验、 新奇抑制摄食实验及强迫游泳实验评估子鼠的抑郁样行为。采用氨基酸分析仪检 测子鼠小肠中氨基酸的变化, 通过蛋白质印迹法检测子鼠肠道中性氨基酸转运蛋 白 ASCT2、 $\mathrm{B}^{0} \mathrm{AT} 1$ 和LAT1的表达。结果: 与对照组比较, 母婴分离组子鼠的体重在出 生后第 21 天和 28 天减轻 $(t=4.925$ 和 5.766 , 均 $P<0.01)$, 糖水偏好百分比减小 $(t=2.709, P<0.05)$, 摄食潜伏期延长 $(t=-13.431, P<0.01)$, 强迫游泳实验中的不动 时间延长 $(t=-3.616, P<0.01)$ 。与对照组比较, 母婴分离组子鼠小肠中的天冬氨酸 浓度增加 $(t=-6.672, P<0.01)$, 谷氨酰胺和甘氨酸浓度减小 $(t=3.107$ 和 9.781 , 均 $P<0.01)$, 同时 ASCT2 和 $\mathrm{B}^{0} \mathrm{AT} 1$ 蛋白表达减少 $(t=6.734$ 和 9.015 , 均 $P<0.01)$, 而 LAT1 蛋白表达增加 $(t=-8.942, P<0.01)$ 。结论: 母婴分离诱导子鼠产生抑郁样 行为, 同时其小肠氨基酸浓度发生变化, 肠道氨基酸转运体表达改变, 提示肠道氨 基酸功能失调与母婴分离诱导的抑郁样行为可能相关。

[关键词 ] 母婴分离; 抑郁; 小肠;氨基酸;转运蛋白; $\mathrm{SD}$ 大鼠

[中图分类号 ］ R2363 [ 文献标志码 ］A

\section{Disorder of intestinal amino acid pathway in depression-like offspring rats induced by maternal separation}

ZHANG Yurong ${ }^{1}$, WANG Ruizhong ${ }^{2}$, WANG Li $^{1}$, CHEN Rui ${ }^{1}$ (1. Section of Scientific Research, the First Affiliated Hospital of Xi' an Medical College, Xi'an 710077, China; 2. Preparation Center, Xi'an Daxing Hospital, Xi'an 710076, China)

Corresponding author: WANG Li, E-mail: 394004312@qq.com, https://orcid.org/0000-

收稿日期: 2020-09-30 接受日期:2020-11-20 网络预发表日期:2021-04-20

基金项目:西安市科技计划 [2019114613YX001SF040(7)]

第一作者:张玉荣,主管药师,主要从事围产期应激与子代情绪障碍的基础研究;E-mail:zyr876281137@126.com; https :// orcid.org/0000-0002-9500-5984

通信作者:王 莉, 教授, 主要从事天然药物临床和基础研究;E-mail :394004312@qq.com; https ://orcid.org/0000-00017401-8906 
[ Abstract] Objective: To investigate the intestinal amino acids pathway in depression-like offspring rats induced by maternal separation. Methods: Sprague-Dawley (SD) female rats were randomly divided into a control group $(n=8)$ and a maternal separation group $(n=8)$. After normal delivery, the maternal rats were separated from offsprings for 14 consecutive days and $3 \mathrm{~h}$ per day in maternal separation group; while rats in the control group was received no interventions in postpartum. Depression-like behaviors of offspring rats were evaluated using the sucrose preference test, novelty suppressed feeding test, and forced swimming test. Amino acid analyzer was used to detect the changes of amino acid contents in the small intestine, and the expressions of alanineserine-cysteine transporter 2 (ASCT2), solute carrier superfamily 6 member 19 (B ${ }^{0}$ AT1) and L-type amino acid transporter 1(LAT1) were detected by Western blot. Results: The weight of the offspring rats in the maternal separation group was significantly lower than that of the control group at 21 and $28 \mathrm{~d}(t=4.925$ and 5.766, all $P<0.01)$. Compared with the control group, the percentage of sucrose preference of the offspring rats in the maternal separation group was significantly reduced $(t=2.709, P<0.05)$, and the feeding latency was significantly prolonged $(t=-13.431, P<0.01)$. The immobility time in FST of maternal separation group was significantly longer $(t=-3.616, P<0.01)$.Increased concentration of aspartic acid $(t=-6.672, P<0.01)$ and down-regulation of glutamine $(t=3.107, P<0.01)$ and glycine $(t=9.781, P<0.01)$ were observed in maternal separation group. Western blot analysis revealed that the protein expressions of ASCT2 $(t=6.734$, $P<0.01)$ and $\mathrm{B}^{0} \mathrm{AT} 1(t=9.015, P<0.01)$ in maternal separation group were reduced, while the expression of LAT1 was increased $(t=-8.942, P<0.01)$. Conclusion: Maternal separation can induce the depression-like behavior in offspring rats; the amino acid contents and the amino acid transporter expression in the small intestine are reduced, which may be related to depression-like behavior induced by maternal separation.

[ Key words ] Maternal separation; Depression; Small intestinal; Amino acid; Transporter; SD rat

[J Zhejiang Univ (Med Sci), 2021, 50(3): 298-304.]

[ 缩略语] 丙氨酸-丝氨酸-半胱氨酸转运蛋白 (alanine-serine-cysteine transporter, ASCT); 溶质载体超家族6成员 19 (solute carrier superfamily 6 member 19,SLC6A19， $\left.\mathrm{B}^{0} \mathrm{AT} 1\right)$; L 型氨基酸转运蛋白 (L-type amino acid transporter, LAT); 放射免疫沉淀法 (radio immunoprecipitation assay, RIPA); 聚偏二氟乙烯 (polyvinylidene fluoride, PVDF); $\mathrm{N}$-甲基-D-天冬氨酸( N-methyl-D-aspartate, NMDA)

抑郁症是全球最常见的精神疾病之一, 具有 易反复发作、发病率高等特点 ${ }^{[1]}$ 。据世界卫生组 织预测, 到 2030 年抑郁症将变成全球首要的致残

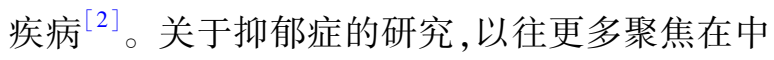
枢神经系统, 而 Gianaros 等 ${ }^{[3]}$ 研究认为抑郁症不
只是单纯的脑功能受损, 更可能出现全身性临床 表现, 如体重下降、消化紊乱及易疲劳等 ${ }^{[4]}$ 。因 此, 外周系统功能紊乱可能成为探索抑郁症发病 的一个途径。有研究发现, 慢性不可预知性温 和刺激模型诱导的抑郁大鼠小肠中氨基酸代谢 
紊乱, 其中谷氨酰胺和甘氨酸的浓度降低, 天冬氨 酸浓度升高, 同时中性氨基酸转运蛋白ASCT2、 LAT 1 和 $\mathrm{B}^{0} \mathrm{AT} 1$ 也发生了变化 ${ }^{[5]}$ 。Rhee 等 ${ }^{[6]}$ 在 2009 年首次提出肠-脑轴, 认为肠道和大脑存在复 杂的相互作用, 大脑的情感中心与肠道功能关联, 抑郁症和胃肠疾病可能有较高的共发率 ${ }^{[7]}$ 。早期 生活应激事件(如母爱剥夺、父母离异或忽视等) 可使成年后生命个体精神类疾病的发病率增加, 如抑郁、焦虑、认知功能障碍等 ${ }^{[8-9]}$ 。经历过早年 虐待的人群产生抑郁的比例高达 $67 \%{ }^{[10]}$ 。因此, 探讨子代抑郁行为的发生意义重大。本研究通过 母婴分离建立子鼠抑郁模型, 观察子鼠的体重及 行为学的变化, 检测子鼠小肠组织中氨基酸浓度 及相关中性氨基酸转运蛋白的改变, 进一步阐明 母婴分离诱导的子代抑郁样行为与肠道氨基酸功 能的关系, 从而为母婴分离诱导子代抑郁的干预 提供理论依据。

\section{1 材料与方法}

\section{1 实验动物和材料}

SD 健康雌性大鼠(240 260 g) 20只由西安交 通大学医学院动物中心提供。动物福利和所有 实验方案均按照我国《实验动物管理条例》进 行。磺基水杨酸为上海邦景实业有限公司产品; L-8900型高速全自动氨基酸分析仪为日本日立高 新技术公司产品; RIPA裂解液为康为世纪公司产 品; $5 \times$ 上样缓冲液为西安晶彩生物科技有限公司 产品; 制胶试剂盒为上海索莱宝生物科技有限公 司产品; 化学发光试剂盒为美国 Millipore公司产 品; ASCT2 抗体、LAT 1 抗体和 $\mathrm{B}^{0}$ AT 1 抗体为美 国 Abcam 公司产品; $\beta$-actin 抗体为陕西先锋生物 科技有限公司产品。

\section{2 建立子鼠抑郁模型}

通过母婴早期分离诱导子鼠抑郁 ${ }^{[11]}$ 。将母 鼠随机分为对照组和母婴分离组各 8 只, 受孕后 单笼喂养。子鼠出生当天为第 0 天, 母婴分离组分 别于第 1 14 天进行母鼠与子鼠分离, 每天将子鼠 移至孵育箱分离 $3 \mathrm{~h}$, 连续 $14 \mathrm{~d}$ 。对照组不进行任 何干预。实验期间正常喂养, 自由饮食饮水。从 子鼠出生至实验结束, 每周测定子鼠的体重。

\section{3 子鼠行为学实验}

1.3 .1 糖水偏好实验测量子鼠的快感缺乏行 为 ${ }^{[12]}$ 给予子鼠 $1 \%$ 蔗糖溶液和自来水, 适应
$24 \mathrm{~h}$ 。第 2 天, 在实验前子鼠禁食禁水 $3 \mathrm{~h}$, 向每笼 大鼠提供 1 瓶 $1 \%$ 蔗糖溶液和 1 瓶自来水 (体积相 近), $1 \mathrm{~h}$ 后称量并记录蔗糖和自来水的消耗量。将 蔗糖和自来水对调位置, $1 \mathrm{~h}$ 后称量并记录其各自 的消耗量。糖水偏好百分比 $(\%)=$ 蔗糖溶液消耗 量/(自来水消耗量+蔗糖溶液消耗量 $) \times 100 \%$ 。

1.3.2 新奇抑制摄食实验评估子鼠的行为紧张 度 ${ }^{[13]}$ 将子鼠放人 $42 \mathrm{~cm} \times 31 \mathrm{~cm} \times 20 \mathrm{~cm}$ 的实验 箱中。在箱子中央的白纸上放一粒鼠粮, 将禁食 $24 \mathrm{~h}$ 的子鼠单独放于箱子的角落。记录子鼠第一 次吃到鼠粮的时间 (即摄食潜伏期), 记录时间为 5 min。

1.3.3 强迫游泳实验测量子鼠的绝望状态 ${ }^{[14-15]}$ 新奇抑制摄食实验结束后, 将子鼠置于高 $50 \mathrm{~cm}$ 、 直径 $20 \mathrm{~cm}$ 、水深 $40 \mathrm{~cm}$ 的圆柱形塑料桶中, 水温 为 $24{ }^{\circ} \mathrm{C}$ 左右。摄像机拍摄 $6 \mathrm{~min}$, 记录后 $4 \mathrm{~min}$ 内 子鼠在水中的不动时间。

1.4 高速全自动氨基酸分析仪检测子鼠小肠组 织中 20 种氨基酸浓度

行为学实验结束后,在子鼠腹腔注射 $20 \%$ 氨 基甲酸乙酯 $(1 \sim 2 \mathrm{~mL} / 100 \mathrm{~g})$ 进行麻醉, 解剖收集远 端小肠组织, 于 $-80^{\circ} \mathrm{C}$ 冰箱保存备用。精确称量一 段远端小肠组织,加入 10 倍量的等渗氯化钠溶液 充分匀浆, 吸取匀浆液, 按体积比 $1: 1$ 加入 $5 \%$ 磺 基水杨酸, 振荡混匀, 离心, 汲取上清液, 用高速全 自动氨基酸分析仪测定其中 20 种氨基酸的浓度。

1.5 蛋白质印迹法检测小肠组织中 ASCT2、 $\mathrm{B}^{0} \mathrm{AT} 1$ 、LAT1 蛋白表达

根据 RIPA 裂解液试剂盒说明书提取子鼠小 肠蛋白质, 定量, 然后加人 $5 \times$ 上样缓冲液, 煮沸变 性3 5 min。根据制胶试剂盒配制聚丙烯酰胺凝 胶电泳的 $10 \%$ 凝胶, 加样。溴酚蓝指示剂显示距 离分离胶底部约 $2 \mathrm{~cm}$ 时停止电泳, 根据标准带指 示进行裁胶, 然后转移至 PVDF 膜上, PVDF 膜用 脱脂奶粉封闭 $2 \mathrm{~h}$,与 $\operatorname{ASCT} 2(1: 1000) 、 \mathrm{~B}^{0} \mathrm{AT} 1$ (1:500)、LAT1 $(1: 1000)$ 抗体 $4{ }^{\circ} \mathrm{C}$ 过夜孵育。 次日, 所有膜清洗后, 与辣根过氧化物酶标记的二 抗进行孵育。最后用化学发光试剂盒发光显色。 用Quantity One 软件对蛋白条带进行定量, 以各条 带与内参 $\beta$-actin 的比值作为蛋白相对表达量。

\section{6 统计学方法}

采用SPSS 18.0 软件进行统计分析。计量数 据采用均数 \pm 标准差 $(\bar{x} \pm s)$ 表示, 组间比较采用 
独立样本 $t$ 检验, $P<0.05$ 为差异有统计学意义。

\section{2 结 果}

2.1 母婴分离对子鼠体重和行为学实验结果的 影响

对照组子鼠出生时 (第 0 天) 平均体重为 $(5.15 \pm 0.44) \mathrm{g}$, 第7、14、21、28天子鼠的体重分别 为 $(16.47 \pm 0.85) 、(30.04 \pm 1.88) 、(50.63 \pm$ $1.85) 、(91.63 \pm 2.13) \mathrm{g}$; 母婴分离组子鼠出生时 (第 0 天)平均体重为 $(5.01 \pm 0.27) \mathrm{g}$, 第 $7 、 14 、 21$ 、 28 天子鼠体重分别为 $(16.38 \pm 0.63) 、(28.62 \pm 2.22)$ 、 $(46.12 \pm 1.81) 、(84.87 \pm 2.53) \mathrm{g}$, 其中第 21 和 28 天体重较对照组减轻 $(t=4.925$ 和 5.766 , 均 $P<0.01)$ 。结果提示, 母婴分离导致子鼠体重减 轻、生长缓慢。

糖水偏好实验结果显示, 对照组子鼠糖水偏 好百分比为 $(70.37 \pm 11.27) \%$, 而母婴分离组为 $(54.88 \pm 11.61) \%$, 差异有统计学意义 $(t=2.709$, $P<0.05)$ 。新奇抑制摄食实验中, 对照组子鼠摄食 潜伏期为 $(98.62 \pm 11.88) \mathrm{s}$, 而母婴分离组摄食潜 伏期为 $(188.13 \pm 14.63) \mathrm{s}$, 两者差异有统计学意 义 $(t=-13.431, P<0.01)$ 。强迫游泳实验中, 对照 组子鼠不动时间为 $(136.88 \pm 19.07) \mathrm{s}$, 而母婴分 离组为 $(181.25 \pm 29.00) \mathrm{s}$, 两者差异有统计学意
义 $(t=-3.616, P<0.01)$ 。结果提示, 母婴分离诱导 子鼠产生抑有样行为。

2.2 母婴分离对子鼠小肠中 20 种氨基酸浓度的 影响

与对照组比较, 母婴分离组子鼠小肠中天冬 氨酸浓度增加 $(P<0.01)$, 谷氨酰胺和甘氨酸浓度 减小 $($ 均 $P<0.01)$, 见表 1 。结果提示, 母婴分离导 致子鼠小肠中氨基酸浓度改变。

2.3 母婴分离对子鼠小肠中氨基酸转运蛋白表 达的影响

对照组 ASCT2、 ${ }^{0}$ AT1、LAT1 蛋白相对表达 水平分别为 $1.11 \pm 0.20 、 1.17 \pm 0.13 、 0.29 \pm$ 0.09 。而母婴分离组 ASCT2、 $\mathrm{B}^{0} \mathrm{AT} 1$ 蛋白相对表 达水平分别为 $0.60 \pm 0.08 、 0.69 \pm 0.08$, 较对照组 减少 $(t=6.734 、 9.015$, 均 $P<0.01)$; LAT1蛋白相 对表达水平为 $0.82 \pm 0.14$, 较对照组增加 $(t=$ $-8.942, P<0.01)$, 见图 1 。结果提示, 母婴分离可 改变子鼠小肠中氨基酸转运蛋白的表达。

\section{3 讨 论}

抑郁症是目前较常见的情绪综合障碍的体 现, 临床以情绪低落、行为迟缓、意志力减退、食欲 低下、体重下降等为典型症状。早期的不良经历 会影响后期的行为、认知等发育过程,也使生长发

表 1 母婴分离对子鼠小肠中氨基酸浓度的影响

Table 1 Effect of maternal separation on the concentration of amino acids in the small intestine of offspring rats

$(\bar{x} \pm s, \mathrm{ng} / \mathrm{mg})$

\begin{tabular}{|c|c|c|c|c|c|c|c|c|}
\hline 组 别 & $n$ & 天冬氨酸 & 谷氨酸 & 天冬酰胺 & 丝氨酸 & 谷氨酰胺 & 苏氨酸 & 精氨酸 \\
\hline 母婴分离组 & 8 & $129 \pm 13$ & $107 \pm 18$ & $365 \pm 14$ & $522 \pm 22$ & $138 \pm 18$ & $480 \pm 31$ & $1470 \pm 227$ \\
\hline 对照组 & 8 & $81 \pm 16$ & $120 \pm 8$ & $379 \pm 16$ & $545 \pm 32$ & $179 \pm 32$ & $508 \pm 21$ & $1676 \pm 162$ \\
\hline$t$ 值 & - & -6.672 & 1.848 & 1.843 & 1.675 & 3. 107 & 2.081 & 2.095 \\
\hline$P$ 值 & - & $<0.01$ & $>0.05$ & $>0.05$ & $>0.05$ & $<0.01$ & $>0.05$ & $>0.05$ \\
\hline 组 别 & $n$ & 丙氨酸 & 脯氨酸 & 甘氨酸 & 缬氨酸 & 蛋氨酸 & 异亮氨酸 & 亮氨酸 \\
\hline 母婴分离组 & 8 & $841 \pm 18$ & $3525 \pm 246$ & $270 \pm 21$ & $632 \pm 36$ & $213 \pm 23$ & $403 \pm 39$ & $804 \pm 24$ \\
\hline 对照组 & 8 & $865 \pm 28$ & $3470 \pm 271$ & $376 \pm 22$ & $663 \pm 24$ & $231 \pm 16$ & $436 \pm 31$ & $785 \pm 17$ \\
\hline$t$ 值 & - & 2. 018 & -0.425 & 9.781 & 2. 024 & 1.797 & 1.837 & -1.805 \\
\hline$P$ 值 & - & $>0.05$ & $>0.05$ & $<0.01$ & $>0.05$ & $>0.05$ & $>0.05$ & $>0.05$ \\
\hline 组 别 & $n$ & 苯丙氨酸 & 赖氨酸 & 组氨酸 & 酪氨酸 & 色氨酸 & 半胱氨酸 & \\
\hline 母婴分离组 & 8 & $432 \pm 34$ & $161 \pm 9$ & $30.6 \pm 2.8$ & $51.9 \pm 2.4$ & $51 \pm 4$ & $147 \pm 6$ & \\
\hline 对照组 & 8 & $459 \pm 20$ & $155 \pm 9$ & $33.9 \pm 3.3$ & $54.8 \pm 3.7$ & $47 \pm 4$ & $142 \pm 5$ & \\
\hline$t$ 值 & - & 1.952 & -1.305 & 2.125 & 1.901 & -2.066 & -2.001 & \\
\hline$P$ 值 & - & $>0.05$ & $>0.05$ & $>0.05$ & $>0.05$ & $>0.05$ & $>0.05$ & \\
\hline
\end{tabular}




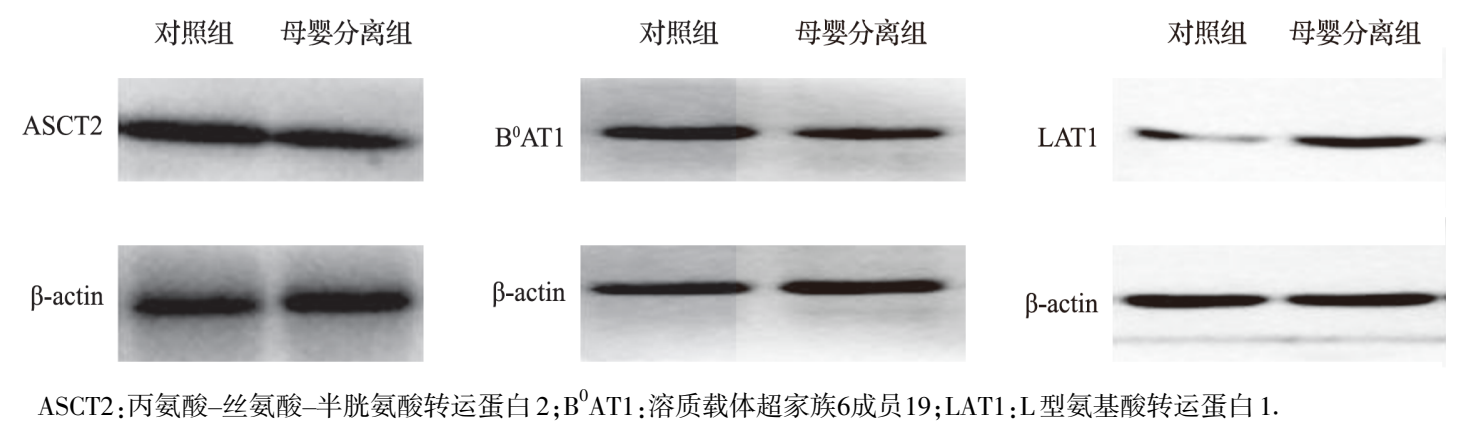

图 1 子代大鼠小肠中 ASCT2、LAT1、 $\mathrm{B}^{0} \mathrm{AT} 1$ 蛋白表达电泳图

Figure 1 Effects of maternal separation on the expression of ASCT2, LAT1, B ${ }^{0}$ AT1 in the small intestine of offspring rats

育较同年龄段缓慢。本研究采用经典的母婴分离 模型,模拟生命早期生活压力下幼儿的成长发育 环境。动物模型研究结果显示, 经历长期母婴分 离应激可使大鼠的体重生长发育落后于同龄大 鼠 ${ }^{[16-17]}$ 。然而,也有研究表明, 子鼠出生后前两周 经历母婴分离可能不会影响其体重 ${ }^{[18]}$, 但青春期 母婴分离会刺激其中脑边缘多巴胺功能发生紊 乱, 对食物产生快感缺乏, 引起体重减轻 ${ }^{[19]}$ 。这 可能与动物的种类、食物刺激等因素有关。本文 资料显示, 经历母婴分离应激可使子鼠的体重较 同期子鼠减轻、糖水偏好百分比减小、快感缺失。 新奇摄食抑制实验用于评估大鼠在新环境中的主 动摄食及行为紧张度, 抑郁会延长摄食潜伏期, 母 紧分离子鼠摄食潜伏期延长。强迫游泳实验中, 母婴分离子鼠不动时间延长, 表现出抑郁状态下 的绝望行为。上述结果表明母婴分离诱导 1 月龄 子鼠产生抑有样行为。

抑有是全身代谢紊乱的疾病 ${ }^{[20]}$, 以往研究多 关注中枢神经系统的氨基酸代谢紊乱 ${ }^{[21]}$, 而氨 基酸的主要来源和吸收的主要部位是小肠 ${ }^{[22]}$ 。

ASCT2、LAT 1、 $\mathrm{B}^{0} \mathrm{AT} 1$ 参与小肠谷氨酰胺和甘氨 酸的摄取转运过程。 $\mathrm{B}^{0} \mathrm{AT} 1$ 和 ASCT2 可吸收饮食 中的谷氨酰胺 ${ }^{[23]}$, 有研究发现小鼠小肠中缺失 $\mathrm{B}^{0} \mathrm{AT} 1$ 可清除谷氨酰胺钠的依赖性摄取, 同时可 减少近 50\%葡萄糖的摄人, 体重也有所下降 ${ }^{[24]}$ 。 本研究中, 母婴分离后子鼠体重的降低很可能 与 $\mathrm{B}^{0} \mathrm{AT} 1$ 的低表达有关。ASCT2 的表达常见于肠 上皮细胞 ${ }^{[25]}$, 可介导谷氨酰胺的交换摄取过 程 ${ }^{[26]}$ 。LAT1 可置换膜表面的部分氨基酸, 如谷氨 酰胺 ${ }^{[27]}$ 。母婴分离的子鼠 LAT1 表达升高, 可能 会降低小肠对谷氨酰胺的摄取。甘氨酸由 $\mathrm{B}^{0} \mathrm{AT} 1$ 在肠黏膜上皮细胞中转运, 通过 ASCT2 在空肠中
介导甘氨酸由顶端膜进入 ${ }^{[28]}$ 。有研究结果显示, 在慢性不可预知性温和应激大鼠抑郁模型中,大 鼠海马组织的甘氨酸水平降低, 尿液中损失增加, 可能缘于其小肠吸收减少 ${ }^{[5]}$ 。本研究发现了小肠 中天冬氨酸浓度增加, 但未进一步证明其转运通 路的变化过程。有研究发现天冬氨酸是一种介导 肠神经调节的调节剂, 可能通过 NMDA 受体的激 活来调节肠道功能 ${ }^{[29]}$ 。因此推测, 天冬氨酸可能 通过 NMDA 受体神经信号通路来介导抑有症的肠 道症状。以往的研究大多是围绕慢性不可预知性 温和刺激或单纯应激导致的抑郁, 研究对象主要 是本代大鼠。为了拓宽多种模型下对抑郁状态全 身氨基酸代谢的认识, 本研究设计母婴分离模型 诱导子鼠产生抑郁, 探索母婴分离诱导对子鼠肠 道氨基酸功能的影响。结果显示, 母婴分离诱导 子鼠小肠中的天冬氨酸浓度升高, 谷氨酰胺和甘 氨酸浓度降低, 进一步检测相应转运蛋白的表达 发现ASCT2 和 $\mathrm{B}^{0} \mathrm{AT} 1$ 表达水平降低,而 LAT1 的表 达升高。

综上,母婴分离可诱导子鼠产生抑郁行为,同 时其小肠氨基酸浓度发生变化,肠道氨基酸转运 蛋白表达改变, 提示肠道氨基酸功能失调与母婴 分离诱导的抑郁样行为可能相关, 但其机制有待 进一步研究探明。

\section{利益冲突 所有作者均声明不存在利益冲突}

[编者按]近年来流行病学研究认为早期生活应激 事件可使成年后个体精神类疾病的发病率增加, 比如抑郁、焦虑、认知功能障碍等。本文采用母 婴分离模型研究子代大鼠的抑郁样行为, 探讨母 婴分离是否导致子代抑郁和焦虑样行为, 以及影 响小肠内氨基酸浓度及转运功能的变化, 从肠一脑 
轴角度探讨发病机制, 有较好的学术意义。研究 结果显示小肠天冬氨酸浓度增高, 谷氨酰胺和甘 氨酸浓度降低, 表明肠道氨基酸功能失调可能与 抑郁样行为相关; 提示今后对子代抑郁的相关氨 基酸千预治疗可能是一个研究思路。当然, 该研 究尚不能明确肠道中性氨基酸转运体ASCT2、 $\mathrm{B}^{0} \mathrm{AT} 1$ 、 LAT1蛋白及氨基酸浓度等在肠一脑轴中 的作用机制, 下一步可以通过相关蛋白的上调或 下调研究, 以及探讨与肠道微生态及短链脂肪酸 的表达关系等, 进一步验证氨基酸转运体在母婴 分离导致子代抑郁和焦虑样行为中的肠一脑轴作 用机制。

\section{参考文献}

[1] WHITEFORD H A, DEGENHARDT L, REHM J, et al. Global burden of disease attributable to mental and substance use disorders: findings from the Global Burden of Disease Study 2010[J]. Lancet, 2013, 382(9904): 1575-1586.

[2] MATHERS C D, LONCAR D. Projections of global mortality and burden of disease from 2002 to 2030 [J/OL]. PLoS Med, 2006, 3(11): e442.

[3] GIANAROS P J, WAGER T D. Brain-body pathways linking psychological stress and physical Health $[\mathrm{J}]$. Curr Dir Psychol Sci, 2015, 24(4): 313-321.

[4] SIMON G E, VONKORFF M, PICCINELLI M, et al. An international study of the relation between somatic symptoms and depression[J]. N Engl J Med, 1999, 341(18): 1329-1335.

[5] YANG X, WANG G, GONG X, et al. Effects of chronic stress on intestinal amino acid pathways $[\mathrm{J}]$. Physiol Behav, 2019, 204(15): 199-209.

[6] RHEE S H, POTHOULAKIS C, MAYER E A. Principles and clinical implications of the brain-gutenteric microbiota axis $[\mathrm{J}]$. Nat Rev Gastroenterol Hepatol, 2009, 6(5): 306-314.

[7] LUKAS V O, KOEN D, JAN T, et al. Central nervous system involvement in functional gastrointestinal disorders $[\mathrm{J}]$. Best Pract Res Clin Gastroenterol, 2004, 18(4): 663-680.

[8] GOODMAN J B, FREEMAN E E, CHALMERS K A. The relationship between early life stress and working memory in adulthood: A systematic review and metaanalysis[J]. Memory, 2019, 27(6): 868-880.

[9] PARK S W, SEO M K, LEE J G, et al. Effects of maternal separation and antidepressant drug on epigenetic regulation of the brain-derived neurotrophic factor exon I promoter in the adult rat hippocampus $[\mathrm{J}]$. Psychiatry Clin Neurosci, 2018, 72(4): 255-265.
[10 ] ANDERSEN S L. Exposure to early adversity: points of crossspecies translation that can lead to improved understanding of depression $[\mathrm{J}]$. Dev Psychopathol, 2015, 27(2): 477-491.

１11］张玉荣, 王瑞忠, 赵 阳, 等. 干酪乳杆菌对母婴分 离诱导产后抑郁大鼠抑郁样行为及海马 NR1 和 $\mathrm{NR} 2 \mathrm{~A}$ 受体表达的影响 $[\mathrm{J}]$. 中华行为医学与脑 科学, 2019, 28(10): 903-907.

ZHANG Yurong, WANG Ruizhong, ZHAO Yang, et al. Effect of Lactobacillus casei on the depressive behavior and the expression of NR1 and NR2A receptors in hippocampus of rats with postpartum depression induced by maternal separation $[\mathrm{J}]$. Chinese Joural of Bahavioral Medicine and Brain Science, 2019, 28(10): 903-907. (in Chinese)

[12] LIU M Y, YIN C Y, ZHU L J, et al. Sucrose preference test for measurement of stress-induced anhedonia in mice[J]. Nat Protoc, 2018, 13(7): 1686-1698.

[13] IBARGUEN-VARGAS Y, SURGET A, TOUMA C, et al. Multifaceted strain-specific effects in a mouse model of depression and of antidepressant reversal $[\mathrm{J}]$. Psychoneuroendocrinology, 2008, 33(10): $1357-$ 1368.

[14] YANKELEVITCH-YABAV R, FRANKO M, HULY A, et al. The forced swim test as a model of depressivelike behavior [J]. J Vis Exp, 2015, (97): 52587.

[15] BAKHTIARZADEH F, NAHAVANDI A, GOUDARZI $\mathrm{M}$, et al. Axonal transport proteins and depressive like behavior, following chronic unpredictable mild stress in male rat[J]. Physiol \& Behav, 2018, 194: 9-14.

[16］郑 玉, 周芩稷, 危智盛, 等. 母紧分离对大鼠成年 后抑郁行为及海马 Gabra6 蛋白表达的影响 $[\mathrm{J}]$. 广 东医学, 2018, 39(23): 3469-3472.

ZHENG Yu, ZHOU Cenji, WEI Zhisheng, et al. Effect of maternal separation on depressive behavior and hippocampal Gabra6 protein expression in rats after adulthood $[\mathrm{J}]$. Guangdong Medical Journal, 2018, 39(23): 3469-3472. (in Chinese)

[17］曹可润. 四逆散对母婴分离抑郁模型大鼠行为学和 5-HT1AR/CREB/BDNF 通路的影响 [D ]. 广州： 广州中医药大学, 2019.

CAO Kerun. The effect of Sinisan on behavior and 5HT1AR/CREB/BDNF pathway of maternal separation depression model[D]. Guangzhou: Guangzhou University of Chinese Medicine, 2019. (in Chinese)

[18 ] IAWASAKI S, INOUE K, KIRIIKE N, et al. Effect of maternal separation on feeding behavior of rats in later life $[J]$. Physiol Behav, 2000, 70(5): 551-556.

[19] JAHNG J W. An animal model of eating disorders associated with stressful experience in early life $[\mathrm{J}]$. Horm Behav, 2011, 59(2): 213-220.

[20] SHIRAYAMA Y, TAKAHASHI M, OSONE F, et al. Myo-inositol, glutamate, and glutamine in the pre- 
frontal cortex, hippocampus, and amygdala in major depression $[J]$. Biol Psychiatry-Cogn NeuroSci NeuroImaging, 2017, 2(2): 196-204.

[21] YANG P, LI X, NI J, et al. Alterations of amino acid level in depressed rat brain $[\mathrm{J}]$. Korean J Physiol Pharmacol, 2014, 18(5): 371-376.

[22] ANDERSON C M, GRENADE D S, BOLL M, et al. $\mathrm{H}^{+}$/ amino acid transporter 1 (PAT1) is the imino acid carrier: an intestinal nutrient/drug transporter in human and $\operatorname{rat}[\mathrm{J}]$. Gastroenterology, 2004, 127(5): 1410-1422.

[23] BRÖER S. Amino acid transport across mammalian intestinal and renal epithelia[J]. Physiol Rev, 2008, 88(1): 249-286.

[24] BRÖER A, KLINGEL K, KOWALCZUK S, et al. Molecular cloning of mouse amino acid transport system B0, a neutral amino acid transporter related to hartnup disorder[J]. J Biol Chem, 2004, 279(23): 24467-24476.

[25] SUNDARAM U, WISEL S, FROMKES J J. Unique mechanism of inhibition of $\mathrm{Na}^{+}$-amino acid cotransport during chronic ileal inflammation $[\mathbf{J}]$. Am J Physiol, 1998, 275(3): G483-G489.

[26] WANG Q, HARDIE R A, HOY A J, et al. Targeting ASCT2-mediated glutamine uptake blocks prostate cancer growth and tumour development $[\mathrm{J}]$. J Pathol, 2015, 236(3): 278-289.

[27] SINGH N, ECKER G F. Insights into the structure, function, and ligand discovery of the large neutral amino acid transporter 1, LAT1 $[J]$. Int J Mol Sci, 2018, 19(5): 1278.

[28] KANAI Y, HEDIGER M A. The glutamate and neutral amino acid transporter family: physiological and pharmacological implications [J]. Eur J Pharmacol, 2003, 479(1-3): 237-247.

[29] AUTRY A E, ADACHI M, NOSYREVA E, et al. NMDA receptor blockade at rest triggers rapid behavioural antidepressant responses $[\mathrm{J}]$. Nature, 2011, 475(7354): 91-95.

[本文编辑 袁天明 沈 敏]

\section{·学术动态・}

\section{吕志民教授团队揭示肿瘤细胞氧化应激调控的新机制}

2021年5月14日, 浙江大学吕志民教授在《分子细胞》(Molecular Cell) 在线发表了题为 “SUCLA2-coupled regulation of GLS succinylation and activity counteracts oxidative stress in tumor cells” 的论文(https://doi. org/10. 1016/j. molcel. 2021.04.002), 揭示了谷氨酰胺酶(GLS)上的一种新型翻译后修饰——琥珀酰化对于GLS活性的重要调节作用, 并发现肿瘤细胞在氧化应激条件下通过琥珀酰辅酶A合成酶亚基 $\beta$ (SUCLA2)调控GLS的琥珀酰化和酶活性,进而增强谷 氨酰胺代谢水平。该研究强调了小分子代谢物质(如琥珀酰辅酶A)调控线粒体代谢活动和细胞氧化压力的新机制,并为 肿瘤治疗新策略的开发提供新思路。

研究人员发现,GLS在胰腺导管腺癌中呈现高表达,相比于正常细胞,胰腺导管腺癌细胞的生长和存活对于谷氨酰 胺代谢表现出更强的依赖性。重要的是,在GLS上也发现了琥珀酰化修饰,该修饰发生在K311位点,可由琥珀酰辅酶A直 接介导。琥珀酰化修饰促进GLS由单体向有活性的四聚体转换, 从而提高了其催化活性,增强了对谷氨酰胺的分解代 谢。SUCLA2是琥珀酰辅酶A合成酶复合体的一个组分, 可以将琥珀酰辅酶A分解为琥珀酸和辅酶A。研究发现,SUCLA2 可以在线粒体内结合GLS,SUCLA2通过代谢反应降低了GLS周围局部的琥珀酰辅酶A的水平,从而降低了GLS的琥珀酰 化修饰水平。而在氧化压力存在的条件下,激活的p38 MAP激酶介导了SUCLA2 S79位点的磷酸化,从而破坏了SUCLA2 与GLS的相互作用, 使SUCLA2与GLS解离, 导致了GLS周围局部琥珀酰辅酶A水平的升高, 进而增加了GLS琥珀酰化修饰, 促进了其活性。激活的GLS增加谷氨酰胺分解和还原性物质的产生, 从而对抗氧化应激,促进肿瘤细胞存活和肿瘤生 长。临床样本中也发现SUCLA2 pS79与GLS K311琥珀酰化水平呈正相关,且与胰腺导管腺癌患者的临床分期和不良预 后呈正相关。

全营营博士研究生、郭栋博士和林树海教授为论文共同第一作者,研究得到国家自然科学基金、科技部重点研发计 划、浙江省自然科学基金资助。 